\title{
Recovery of Gold
}

EXPERIMENTS WITH A LOW GRADE BENCH SCRAP

\author{
B. Calmanovici, H. Kerbel and L. Gal-Or
}

Center for Noble Metals, Institute of Metals, The Technion, Haifa, Israel

\begin{abstract}
Small scale recovery of gold from various types of waste materials attracts continuing attention. A study of wet recovery of the metal from a low grade bench scrapproduced during jewellery manufacture is described in this article.
\end{abstract}

Mechanical polishing is common practice in jewellery manufacture. The waste thus formed may contain grinding wheel grit and paste, sand paper grit, bench dirt and other contaminants. After burning out to remove the combustible components the remaining material may contain 2 to 3 per cent of gold and an appreciable percentage of iron oxides which are used as abrasive materials. As a result of the large ratio between iron products and gold alloy and the intimate mixing of these components, magnetic separation of the iron is impossible, the entire mixture being affected by the magnet. On a small scale, chemical methods of recovery may therefore be applied $(1,2)$ based on selective dissolution of the non-gold components and/or total dissolution followed by selective precipitation of the gold. Common chemical separation methods include digestion of scrap with nitric acid, diluted with its own volume of water, to dissolve the iron products and the copper, silver and zinc in the gold alloy. This is followed by digestion of the remaining material with aqua regia and precipitation of the gold with a reducing agent such as sodium bisulphite. A simpler procedure may be direct digestion with aqua regia followed by selective gold precipitation.
The purpose of the experiments described below was to compare the percentages and purities in which gold was recovered by the two methods. Experiments were carried out on material obtained from a jewellery manufacturer which had been produced by burning and grinding of a low grade bench scrap. It had a high iron content which could not be separated by use of a magnet.

Two methods for recovery of its gold content were explored: (1) Digestion with a 1:1 mixture of nitric acid and water, followed by digestion of the insoluble residue with aqua regia and precipitation of the gold from the solution thus formed

(2) Direct digestion with aqua regia, followed by precipitation of the gold from the solution obtained.

In each procedure, the digestion steps were repeated a number of times in order to determine the effects, if any, of such repetition on the quantities of gold recovered.

Initial experiments showed that when large concentrations of iron are present in the solution, it can be coprecipitated with the gold by sodium bisulphite. The presence of iron manifests itself by a darkening of the colour of the precipitate, which changes to

Table I

Recovery of Gold after Precipitation from Solutions Obtained by Digestion of a Low Grade Bench Scrap in Aqua Regia With and Without Prior Digestion in 1:1 Nitric Acid

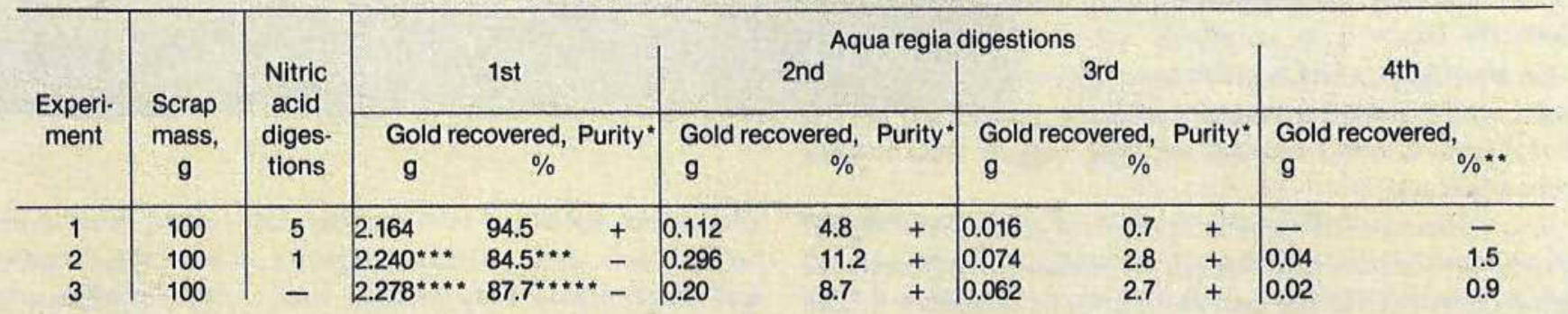

* As judged by the colour of the precipitate and the reaction of the filtrate (after nitric acid wash) with ammonium thiocyanate * Based on total obtained by extrapolation of the data 
normal after washing with 1:1 nitric acid. The presence of iron in solutions of the precipitate was established also by its specific reaction with ammonium thiocyanate. The codeposition of iron with gold indicates a large shift of its deposition potential due to the high concentrations in which it is present. The codeposited iron may be removed by washing the deposit with hot 1:1 nitric acid. However, the iron cannot be dissolved by such nitric acid if the precipitate has been dried beforehand in an oven at elevated temperature $\left(\sim 900^{\circ} \mathrm{C}\right)$. During such treatment, passivation of the iron apparently sets in, making its dissolution impossible.

Table I summarizes the experiments and the results obtained after treating $100 \mathrm{~g}$ samples of scrap with successive digestions in aqua regia. The percentage of gold recovered after each digestion as compared to the total gold content is given in the table.

The following conclusions can be drawn concerning gold recovery from this type of scrap:

Repeated digestions with 1:1 nitric acid result in dissolution of a higher percentage of the gold in the scrap during the first subsequent digestion with aqua regia.

Direct digestion with aqua regia results in codeposition of iron, and probably other metals also, with the gold during the sodium bisulphite precipitation. Furthermore, a lower fraction (88 per cent) of the original gold content is recovered after the first aqua regia digestion. However, it is possible to purify the precipitate by boiling it with 1:1 nitric acid; analysis by atomic absorption spectroscopy showed only 0.3 per cent silver and 0.01 per cent iron to be present in the sample so-treated.

Repeated digestions with aqua regia after repeated earlier digestions with nitric acid (method 1) results in additional recovery of small percentages of gold. In the case of the scrap reported on here the recoveries in three successive digestions were $94.5,4.8$ and 0.7 per cent of the total gold content. In practice, therefore, the cost-effectiveness of additional digestion should be considered.

\section{Acknowledgement}

The authors wish to express their gratitude to Mr. M. Goldwein (Noga Jewellery) who supplied the taw material.

\section{References}

1 R. Loewen, 'Small Scale Gold Refining', Technical report 44/1, Worshipful Company of Goldsmiths Technical Advisory Committee, July, 1980 2 Anon., Gold Bull., 1981, 14, (1), 24

\section{Gold Uses, Ancient and Modern}

In the modern world with its changing technology it is often surprising to find that some gold technology developed in antiquity is still finding application today. A timely reminder of such a use, namely that of gold leaf for decoration, has been discussed in a very recent issue of The Gold News, $(1983,8,(1), 2)$, published under the auspices of the Gold Institute, in which the decoration of the 50 years-old, 26-storey Crown Building in Fifth Avenue, New York City with 23 carat gold leaf is described. The gold has been applied not only to the crown of the building but also to the dormers and the ornamental spandrels beneath 220 windows. An area of just over $2000 \mathrm{~m}^{2}$ of the Crown Building has been covered in gold leaf which, in combination with the illumination designed for it, creates a landmark clearly visible from all directions in the city. It is the largest single application of gold leaf in the U.S.A.

The weather resistant and maintenance-free characteristics of gold leaf ensure retention of its decorative value for many years, and its use appears to be increasing in popularity as a result. It is interesting that in principle the method of manufacture of gold leaf today differs little from that used over the past five millenia viz. the melting of gold to form an ingot which is then hammered or beaten to extraordinary thinness, of the order of one-tenth of a micrometre. The beating is now carried out mechanically in a way designed to imitate as closely as possible the hand hammering of craftsmen from antiquity to relatively recent times. Discovered treasure hoards and manuscripts throughout the ages have revealed a remarkable constancy of the technique and its usage. An instructive account of such examples has been given by E. D. Nicholson, Gold Bull., 1979, 12, (4), 161-166, who describes samples of gold leaf which were found among the treasures of the tomb of Tutankhamen at Luxor, and the inner, gilded coffin case of Henutmehit which is now at the British Museum. The probability of gold beating being practised in India from the birth of Buddhism (5th century B.C.) is discussed as are accounts of the practice in the 12th century A.D. by Theophilus and of that in France in 1763 , by William Lewis.

Interestingly and in complete contrast, in the same issue of The Gold News, an account is given of a contemporary and totally novel use of gold, in an alloy component of Josephson junctions. Such junctions are used as high-speed switches which could well replace existing transistor switches in data processors.

In work carried out at Bell Laboratories, Short Hills, N. J. a working data processor has been built consisting of almost 600 tiny, fast switches, all operating at temperatures of a few degrees above absolute zero and packed on a chip the size of a collar button. The Josephson junctions each consist of a 3-layer sandwich of a gold-indium-lead alloy, an oxide insulating barrier and a top layer of gold-lead alloy, each layer being about a micrometre thick. When operated at low temperatures the junctions switch on and off an order of magnitude faster than the best conventional transistor switches. The connections are superconducting so that the flow of information within the circuit is unhindered. Because Josephson switches emit only a fraction of the heat produced by conventional transistors they can be packed very much closer together with impressive compaction advantages. Uses anticipated for data processors operating with Josephson switches include faster, more efficient image processing, for example in colour television sets, recognition of human speech and meteorological applications.

It is an intriguing facet of gold technology that an application developed 5000 years ago continues to be used at the same time as the most modern electronic developments involving gold and its alloys. CI 